\title{
Tunable surface plasmons in Weyl semimetals TaAs and NbAs
}

\author{
Gennaro Chiarello, ${ }^{1, *}$ Johannes Hofmann, ${ }^{2,3, \dagger}$ Zhilin $\mathrm{Li},{ }^{4}$ Vito Fabio, ${ }^{1}$ Liwei \\ Guo, ${ }^{4}$ Xiaolong Chen, ${ }^{4}$ Sankar Das Sarma, ${ }^{5}$ and Antonio Politano ${ }^{6,7, \ddagger}$ \\ ${ }^{1}$ Department of Physics, University of Calabria, via ponte Bucci, 31/C, 87036 Rende (CS), Italy \\ ${ }^{2}$ Department of Applied Mathematics and Theoretical Physics, University of Cambridge, \\ Centre for Mathematical Sciences, Cambridge CB3 0WA, United Kingdom \\ ${ }^{3}$ TCM Group, Cavendish Laboratory, University of Cambridge, Cambridge CB3 OHE, United Kingdom \\ ${ }^{4}$ Beijing National Laboratory for Condensed Matter Physics, \\ Institute of Physics, Chinese Academy of Sciences, Beijing 100190, China \\ ${ }^{5}$ Condensed Matter Theory Center and Joint Quantum Institute, Department of Physics, \\ University of Maryland, College Park, Maryland 20742-4111 USA \\ ${ }^{6}$ Istituto Italiano di Tecnologia, Graphene Labs, Via Morego,30, 16163 Genova, Italy \\ ${ }^{7}$ Department of Physical and Chemical Sciences, \\ University of L'Aquila, Via Vetoio,67100 L'Aquila, Italy
}

(Dated: November 13, 2018)

\begin{abstract}
By means of high-resolution electron energy loss spectroscopy, we investigate the low-energy excitation spectrum of transition-metal monopnictides hosting Weyl fermions. We observe gapped plasmonic modes in (001)-oriented surfaces of single crystals of NbAs and TaAs at 66 and $68 \mathrm{meV}$, respectively. Our findings are consistent with theory and we estimate an effective Coulomb interaction strength $\alpha_{\text {eff }} \approx 0.41$ for both samples. We also demonstrate that the modification of the surface of transition-metal monopnictides by the adsorption of chemical species (in our case, oxygen and hydrocarbon fragments) changes the frequency of the plasmonic excitations, with a subsequent modification of the effective interaction strength in the $0.30-0.48$ range. The remarkable dependence of plasmonic features on the presence of adsorbates paves the way for plasmonic sensors based on Weyl semimetals operating in the mid-infrared.
\end{abstract}

Massless Dirac fermions, previously observed in two dimensions in graphene [1] and topological insulators [2], have recently been observed in three-dimensional topological Dirac semimetals (TDS) [3-5] and topological Weyl semimetals (TWS) [6]. These three-dimensional semimetals represent a novel state of quantum matter with bulk Weyl fermions and non-trivial topological surface states forming Fermi semi-arcs [7, 8]. The topological bulk and surface band structure of TWS gives rise to a plethora of novel phenomena, such as negative magnetoresistance [9], chiral magnetic effects [10, 11], chiral magnetoresistance [12, 13], and anisotropic Adler-BellJackiw anomaly [14]. The signatures of TWS have been revealed in NbP [15, 16], TaP [16-19], TaAs [20-25], and NbAs [26].

In particular, the use of topological materials has opened new pathways for plasmonics [27]. As the separation of Weyl nodes in both momentum and energy acts as an effective applied magnetic field in momentum space, plasmonic modes in TWS behave similarly to magnetoplasmons in ordinary metals $[28,29]$. The application of magnetic fields also activate further intriguing peculiarities, including coupling-induced transparency and broadband polarization conversion [30]. Therefore, TWS are very interesting candidates for plasmonic devices.

However, to date the use of TWS in plasmonics has been hindered by the absence of samples with high crystalline quality. Specifically, the crystal growth from melt is impeded by the facile sublimation of As before melting and by the presence of other phases in the Ta-As [31] and
$\mathrm{Nb}-\mathrm{As}$ [32] binary system. Moreover, in standard conditions, chemical vapor transport using iodine as transport agent only provides crystals with small size $(0.5-1.5 \mathrm{~mm})$. By using foils instead of powder as the starting material, tilting the ampoule to improve convections, and adjusting the concentration of agent iodine, we were able to optimize the growth and obtain single-crystal foils of transition-metal arsenides in the centimeter scale with unprecedented crystalline quality [33].

In this Letter, we make use of this experimental advance in crystal growth to study the plasmonic spectrum of both NbAs and TaAs single crystals by means of highresolution electron energy loss spectroscopy (HREELS). We find that the plasmon frequency is similar in both compounds independent of the doping level, in agreement with predictions from the high-temperature random phase approximation (RPA). Moreover, we discover that the plasmon frequency can be tuned when chemical species are adsorbed at NbAs and TaAs surfaces, highlighting the high potential of TWS plasmonic modes in the field of sensors.

Our TaAs and NbAs crystals were grown by chemical vapor transport method [33]. In a typical run, tantalum (or niobium), arsenic, and iodine (transport agent, $5 \mathrm{mg} / \mathrm{ml}$ ) were loaded in a silica ampoule under argon. The ampoule was then evacuated, sealed, and heated gradually from room temperature to $1050{ }^{\circ} \mathrm{C}$ over 72 hours. Afterwards, the ampoule was put to a temperature gradient from $1070{ }^{\circ} \mathrm{C}$ to $1030{ }^{\circ} \mathrm{C}$ for two weeks and naturally cooled down to room temperature. As 
shown in Fig. 1, the obtained crystals have regular shapes and shiny facets. Experiments were performed in an ultra-high vacuum (UHV) chamber with a base pressure of $10^{-11}$ mbar. The absence of contaminants has been ensured by means of Auger electron spectroscopy (AES) and vibrational spectroscopy, while X-ray diffraction (XRD) and low-energy electron diffraction (LEED) have been used to study bulk and surface structural order, respectively. All experiments have been carried out at room temperature. Crystals show large-scale terraces (001)-oriented, as also confirmed by XRD (Fig. 1). We chose those surfaces since the Weyl cones in the bulk and the Fermi-arc surface states are observed in the (001)terminated surface on both NbAs [26] and TaAs [20]. Samples have been cleaved in situ in UHV. The experimental crystal structure of NbAs and TaAs is shown as an inset in Fig. 1. In NbAs (TaAs), $\mathrm{Nb}(\mathrm{Ta})$ and $\mathrm{As}$ atoms are six-coordinated to each other. They crystallize in a body-centered tetragonal unit cell with lattice constants $a=3.45 \AA(3.44 \AA)$ and $c=11.68 \AA(11.64 \AA)$. For both samples, the space group is $I 4_{1}$ md (No. 109) and the structure lacks inversion symmetry.

The HREELS excitation spectrum of (001)-oriented pristine NbAs and TaAs single crystals at room temperature in shown in Fig. 2. The experiments were performed with a Delta 0.5 spectrometer by Specs GmbH, Germany, in a specular geometry, i.e., with equal incident and scattered electron beam angles $\alpha_{i}=\alpha_{s}=72^{\circ}$ with respect to the surface normal, with an incident energy $E_{i}=47 \mathrm{eV}$ in order to minimize the inelastic mean free path for electrons [34] and to increase the surface sensitivity by enhancing the spectral weight of surface-related excitations. The loss spectra of both samples exhibit a single peak at a similar energy at $66 \mathrm{meV}$ and $68 \mathrm{meV}$, respectively. The angle-integrated scattering cross section for HREELS [35, 36]

$$
\frac{d \sigma}{d E} \sim \frac{1+n(E)}{E \cos \alpha_{i}} \operatorname{Im}\left[1+\varepsilon\left(E, q_{\|}\right)\right]^{-1},
$$

has a resonance for surface plasmonic modes $1+$ $\varepsilon\left(E, q_{\|}\right)=0[35,36]$. Here, $E$ is the energy loss transferred to the surface, $n(E)$ the Fermi-Dirac distribution, and $q_{\|}$is the parallel momentum transfer [37]:

$$
q_{\|}=\frac{\sqrt{2 m E_{i}}}{\hbar}\left(\sin \alpha_{i}-\sqrt{1-\frac{E}{E_{i}}} \sin \alpha_{s}\right) .
$$

Hence, we identify the peaks at $66 \mathrm{meV}$ and $68 \mathrm{meV}$ with the surface plasmon modes of the TWS. The plasmon frequency is quite similar in both materials, indicating that the Fermi velocity and the interaction strength are of comparable magnitude, consistent with band-structure calculations [38]. Recent optical conductivity measurement on the possible TWS $\mathrm{Eu}_{2} \mathrm{Ir}_{2} \mathrm{O}_{7}$ [39] reported plasmon energies in the $50-90 \mathrm{meV}$ range, quite consistent with our findings for NbAs and TaAs. It is worthwhile pointing out that the observed excitations cannot
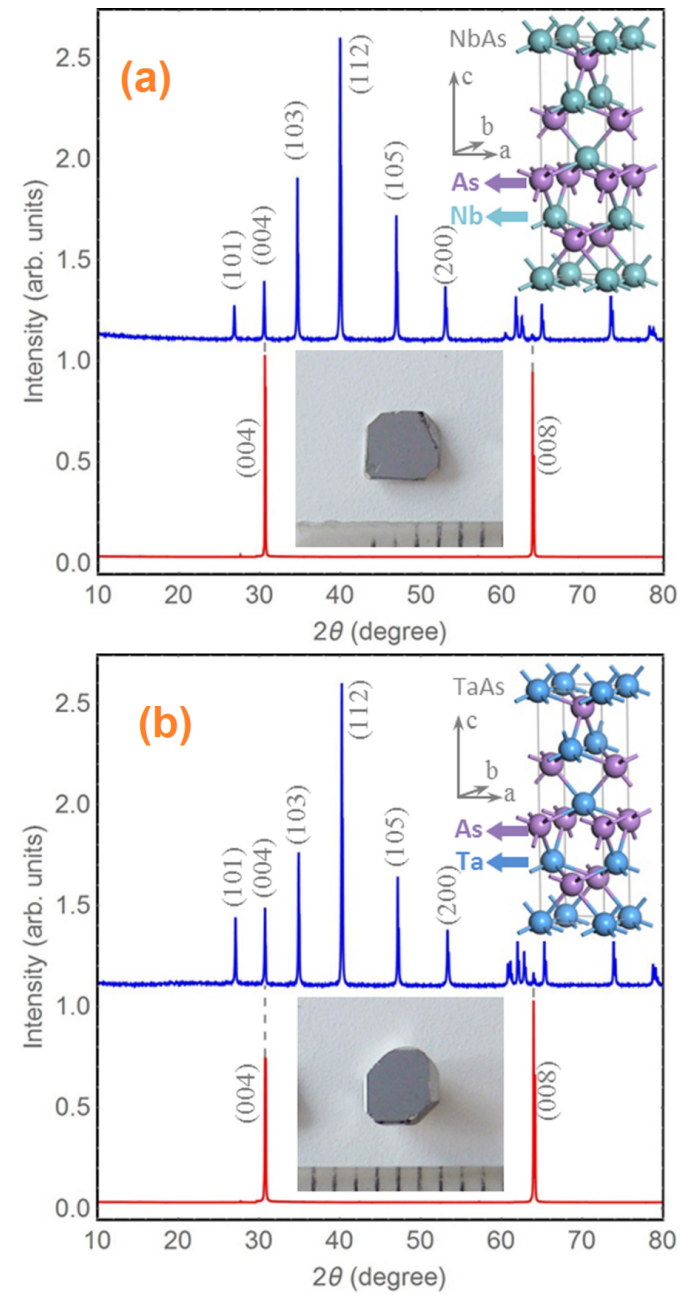

FIG. 1. (a) XRD pattern of the as-grown NbAs crystal $\left(\mathrm{CuK}_{\alpha}\right)$. The top panel shows the pattern for NbAs powder, while the bottom panel is related to the (001)-oriented flat surface of the single crystal. The inset of the top panel shows the crystal structure (purple for As and blue for $\mathrm{Nb}$ ). The bottom panel shows the sample compared with a millimeter ruler. (b) XRD pattern of TaAs (with blue for Ta). The notation is the same as in (a).

be associated to phonons or polaritons. As a matter of fact, composite modes arising from the hybridization of phonon modes with plasmons should have a gap of the order of the highest branch of optical phonons in NbAs (33 meV [40]) and TaAs (32 meV [40, 41]). In the excitation spectrum acquired with a primary electron beam energy of $92 \mathrm{eV}$ (inset of Fig. 2, for the case of NbAs), we observe also other resonances at $1.5,8.8$ and $15.3 \mathrm{eV}$ (inset of Fig. 2). While the first two features are originated by interband transitions, the latter excitation can be attributed to the plasmonic response of large hole pockets [42], consistent with calculations of the plasma edge [43].

The similar values for the plasmon frequency in both samples and the smallness of typical doping 


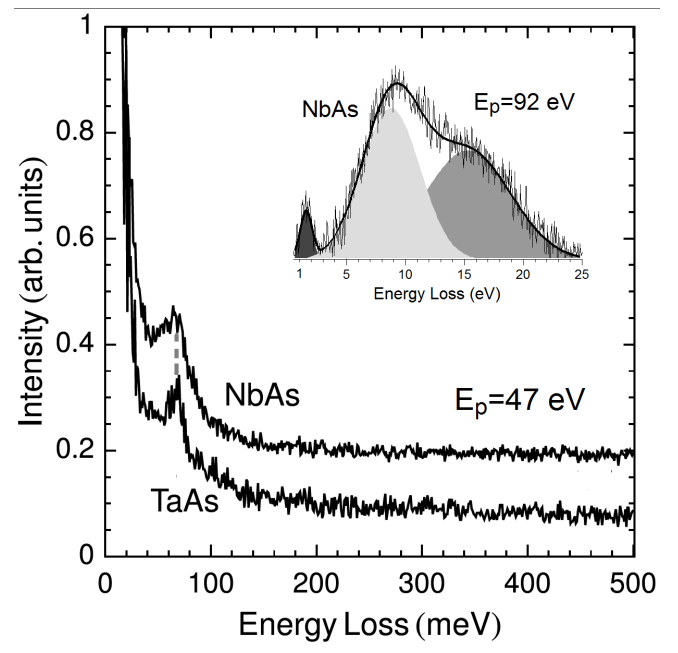

FIG. 2. HREEL spectra of (001)-oriented NbAs and TaAs single crystals at room temperature acquired for an impinging energy of $47 \mathrm{eV}$ in grazing scattering conditions. The spectra are off-set vertically. The position of the plasmon resonance is indicated by the vertical dashed gray line. Note that the excitation spectrum probed by HREELS in the specular geometry does not exhibit the optical phonon modes of NbAs and TaAs at $33 \mathrm{meV}$ [40] and $32 \mathrm{meV}$ [40, 41], respectively, since phonon modes can be excited in HREELS only in the off-specular (impact scattering) geometry [44] The inset shows the excitation spectrum in an extended energy range probed for $\mathrm{NbAs}(001)$ for an impinging energy of $92 \mathrm{eV}$.

levels $(\sim 10 \mathrm{meV} \approx 100 \mathrm{~K}$, close to the charge-neutral point [42]) indicate that we probe the universal hightemperature regime, where the plasmon is formed of thermally excited interband carriers and which is independent of the doping level [45]. Within the RPA, the dielectric function at finite temperature and doping was computed in Ref. [45]. At high temperature, an analytical expression is given by [45]

$$
E_{\text {plas }}=\sqrt{\frac{2 \pi g \alpha}{\kappa(T)}} \frac{T}{3},
$$

where $T$ is the temperature (room temperature in our case), $v_{F}$ is the Fermi velocity, $g$ is the number of Weyl nodes ( $g=24$ here) and $\alpha=e^{2} / \hbar v_{F} \varepsilon_{\infty}$ is the dimensionless Coulomb interaction strength (the WSM "fine structure constant", in analogy with quantum electrodynamics [46]), usually ranging between 0.2 and 2 . In addition, $\kappa(T)$ accounts for the effective temperature-dependent electronic renormalization of the dielectric constant, commonly parametrized in terms of a "Landau pole" scale $\Lambda_{L}$ as $\kappa(T)=\frac{g \alpha}{3 \pi} \ln \frac{\hbar v_{F} \Lambda_{L}}{\pi T e^{-\gamma_{E}}}$, where $\gamma_{E}$ is Euler's constant [45]. The fact that the long-wavelength plasma frequency (3) of WSM depends on the interaction coupling constant and the Landau scale is a direct manifestation of the non-Fermi-liquid nature of intrinsic WSM by virtue of the Weyl point being a quantum critical point [47].
The appearance of the Landau pole is a manifestation of electron interactions in the WSM: while the classical theory of a WSM is scale-invariant (i.e., the singleparticle Dirac Hamiltonian scales in the same way as the Coulomb interaction under a change of length scales), this classical symmetry is broken once quantum effects through the Coulomb interaction are taken into account. The dimensionless coupling $\alpha$ of the classical system now depends on the temperature or doping scale at which it is measured. RPA predicts this "running" of the coupling from weak coupling at small temperatures $T \ll \Lambda_{L}$ to strong coupling as $T$ approaches the Landau scale $\Lambda_{L}$, where the RPA breaks down [46]. The dimensionless coupling constant $\alpha$ is replaced by the dimensional quantity $\Lambda_{T}$, which is known as "dimensional transmutation". For the plasmon (3), dimensional transmutation implies that the naive (by dimensional analysis) linear-in-temperature dependence of the frequency is replaced by a super-linear temperature-scaling that depends on the Landau pole. A fit of the Landau pole (assuming the RPA result) has predictive power for other temperature and density scales, whereas the fine structure constant $\alpha(T)$ is always measured at a fixed temperature.

Both TaAs and NbAs have a total of 24 Weyl nodes, which are divided in two classes W1 and W2 with different band parameters. We fit the peaks in Fig. 2 using the high-temperature expression with $T=300 \mathrm{~K}$. The fit value for the Landau pole is $\Lambda_{L}=126 \pm 35 \mathrm{meV}$ and $\Lambda_{L}=118 \pm 18 \mathrm{meV}$ for NbAs and TaAs, respectively, corresponding to an effective interaction strength of $\alpha_{\text {eff }}=\frac{\alpha}{\kappa(T)}=0.41 \pm 0.07$. This large value of the interaction strength (at room temperature) indicates that the WSMs TaAs and NbAs are not weakly coupled and are no longer described by leading-order perturbation theory in $\alpha$ [46].

Most practical application of TWS should work in ambient conditions. Thus, it is crucial to evaluate the effect of surface modifications on the plasmon frequency. In addition, tunability of surface-plasmon frequency is highly desirable for the prospect of plasmonic sensors [48, 49].

Here, we consider two case-study examples of chemisorbed species providing opposite sign in the shift in the surface-plasmon frequency. Figure 3 shows the behavior of plasmonic modes in TWS samples saturated with oxygen at room temperature (bottom two black and red lines). Being As-terminated, TWS samples are not inert and interact strongly with oxygen [50]. The resulting loss spectrum is dominated by a band centered at $100 \mathrm{meV}$ [51], with the main contribution from the As$\mathrm{O}$ stretching mode and minor spectral contribution from $\mathrm{Nb}$ (Ta)-O stretching [50]. The plasmon frequency is redshifted compared to the pristine crystal to $58 \mathrm{meV}$ in both NbAs and TaAs (solid gray line). This red-shift can be accounted for by a change in the surface dielectric function. The plasmon condition changes to $\varepsilon_{s}(E)+\varepsilon(E)=0$, where the dielectric function $\varepsilon_{s}(E)$ due to adsorbed par- 
ticles becomes [36]

$$
\varepsilon_{s}(E)=1+\frac{4 \pi e^{* 2} n}{M} \frac{1}{E_{T}^{2}-E^{2}-i E \Gamma},
$$

where $E_{T}$ is the bare oscillation frequency of the As$\mathrm{O}$ bond. The adsorbate contributes to the dielectric function with a harmonic oscillator term, with a small (though existing) hybridization between surface collective electronic excitations and the adsorbate mode. Thus, the red-shift can be accounted for by a coupling of the adsorbate layer and the plasmonic mode as:

$$
\varepsilon(E)=\varepsilon_{\infty}\left(1-\frac{E_{p}^{2}}{E(E+i \Gamma)}\right)+\Delta \varepsilon \frac{E_{T}^{2}}{E_{T}^{2}-E^{2}-i E \Gamma^{\prime}} .
$$

A fit to the data gives $\Delta \varepsilon=8 . \varepsilon_{s}(E)$ is positive for $E<E_{T}$ and thus decreases the plasmon frequency. The effective interaction strength is $\alpha=0.30 \pm 0.15$ and $\alpha=$ $0.31 \pm 0.16$ for O-modified NbAs and TaAs, respectively. The Landau pole $\Lambda_{L}$ in the two cases is $165 \pm 108$ and $165 \pm 112 \mathrm{meV}$.

It is also worth mentioning that the shift of the plasmon frequency due to changes in carrier density arising from adsorbate-induced surface doping further supports the interpretation of the modes in pristine NbAs and TaAs (Fig. 1) as surface rather than bulk excitations. Especially, interaction with oxygen has been recently demonstrated only to affect Fermi-arc states [50], while the O-induced modification of the other electronic bands is insignificant.

An opposite change in the plasmon frequency (different from the plasmon-adsorbate hybridization) can arise from an effective charge transfer from adsorbates to the system or vice versa. Such a doping effect plays out when hydrocarbon fragments are present over the surface of the TWS. Their presence is evident in HREELS spectra in Fig. 3 (top two orange and green lines), which display C-H bending and stretching modes at $170 \mathrm{meV}$ and $360 \mathrm{meV}$, respectively $[52,53]$, arising from the decomposition of hydrocarbons over the surfaces of NbAs and TaAs. The plasmon frequency is blue-shifted to $72 \mathrm{meV}$ and $73 \mathrm{meV}$ in NbAs and TaAs, respectively. We ascribe this shift to the electron transfer (n-doping) from $\mathrm{C}-\mathrm{H}$ groups to NbAs and TaAs. The fit gives an effective interaction strength $\alpha=0.48 \pm 0.08$ and $\alpha=0.47 \pm 0.21$ for NbAs and TaAs, respectively. The Landau pole $\Lambda_{L}$ in the two cases is $103 \pm 14$ and $106 \pm 40 \mathrm{meV}$.

Finally, in order to evaluate the feasibility of TWSbased plasmonics it is important to assess the lifetime of the plasmonic modes through the analysis of the linewidth of the HREEL peak. The lifetime of surface collective electronic excitations notably influences plasmonmediated dynamic processes, the field enhancement of surface enhanced Raman scattering (SERS) [54], and surface-enhanced fluorescence [55]. In this case, we only

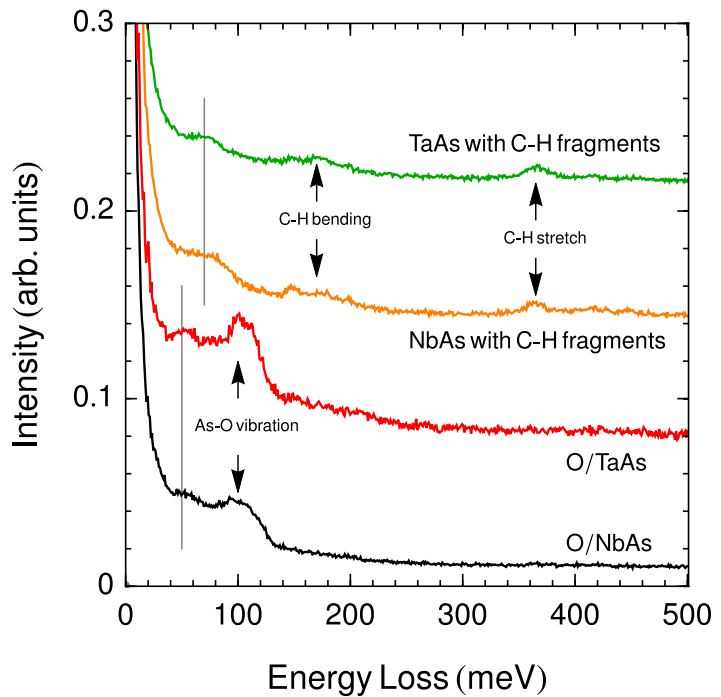

FIG. 3. HREEL spectrum of TaAs and NbAs with C-H fragments (top two solid green and orange lines) and O-modified surfaces (bottom two solid red and black lines). The spectra are off-set vertically. The vertical solid gray lines denote the position of the plasmon resonance, which is red-shifted in O-modified samples compared to the pristine surface, and blue-shifted for samples modified by the adsorption of hydrocarbon fragments. Loss spectra show additional features associated with $\mathrm{C}-\mathrm{H}$ bending and stretching vibrations and As-O vibrational modes, respectively, which are indicated by the arrows.

note a decrease of the lifetime of the plasmonic excitation by less than one half, as chemisorbed species favor phase-breaking scattering events through collisions of electrons with subsequent enhancement of the Landau damping and, consequently, a broadening of the plasmonic peak. However, this increase of the lifetime is relatively low compared to the total quenching induced by chemisorbed species experienced for the case of graphene plasmons [56], in spite of the low chemical reactivity of graphene [57].

In conclusion, we have studied the low-energy excitation spectrum in (001)-oriented NbAs and TaAs surfaces. The loss spectrum measured by HREELS, dominated by surface plasmons in the mid-infrared, has been fitted by RPA model in order to extract both the effective Coulomb interaction strength and the Landau pole. The pristine NbAs and TaAs single crystals are characterized by a single plasmonic resonance at $66 \mathrm{meV}$ and $68 \mathrm{meV}$, respectively. The strong reactivity toward oxygen has noticeable implications on the plasmonic spectrum of TWS, due to the change of the dielectric function induced by oxygen adatoms stably adsorbed at room temperature. The plasmon energy is shifted down to $58 \mathrm{meV}$ for both $\mathrm{NbAs}$ and TaAs, with a subsequent reduction of the effective strength. In the presence of fragments of hydrocarbons, a charge transfer from adsorbates to TWS occurs with a blue-shift of the plasmonic frequency up to 72 and 
$73 \mathrm{meV}$ in NbAs and TaAs, respectively. Our results evidence that the plasmonic features of the pristine NbAs and TaAs surfaces can be preserved only by protecting the active channel of the TWS-based plasmonic device with a capping layer in order to reduce interaction with environmental species. On the other hand, the striking dependence of plasmonic features of TWS on the presence of adsorbates can be used to develop a new generation of plasmonic chemical sensors.

JH acknowledges support by Gonville and Caius College and Peterhouse, Cambridge.

* gennaro.chiarello@fis.unical.it

† jbh38@cam.ac.uk

¥ antonio.politano@iit.it

[1] K. S. Novoselov, A. K. Geim, S. V. Morozov, D. Jiang, M. I. Katsnelson, I. V. Grigorieva, S. V. Dubonos, and A. A. Firsov, "Two-Dimensional Gas of Massless Dirac Fermions in Graphene," Nature 438, 197 (2005).

[2] C. W. Luo, H. J. Wang, S. A. Ku, H.-J. Chen, T. T. Yeh, J.-Y. Lin, K. H. Wu, J. Y. Juang, B. L. Young, T. Kobayashi, C.-M. Cheng, C.-H. Chen, K.-D. Tsuei, R. Sankar, F. C. Chou, K. A. Kokh, O. E. Tereshchenko, E. V. Chulkov, Yu. M. Andreev, and G. D. Gu, "Snapshots of Dirac Fermions near the Dirac Point in Topological Insulators," Nano Letters 13, 5797 (2013).

[3] S. Borisenko, Q. Gibson, D. Evtushinsky, V. Zabolotnyy, B. Büchner, and R. J. Cava, "Experimental Realization of a Three-Dimensional Dirac Semimetal," Phys. Rev. Lett. 113, 027603 (2014).

[4] Z. K. Liu, J. Jiang, B. Zhou, Z. J. Wang, Y. Zhang, H. M. Weng, D. Prabhakaran, S-K. Mo, H. Peng, P. Dudin, T. Kim, M. Hoesch, Z. Fang, X. Dai, Z. X. Shen, D. L. Feng, Z. Hussain, and Y. L. Chen, "A Stable ThreeDimensional Topological Dirac Semimetal $\mathrm{Cd}_{3} \mathrm{As}_{2}$," Nat. Mater. 13, 677 (2014).

[5] Z. K. Liu, B. Zhou, Y. Zhang, Z. J. Wang, H. M. Weng, D. Prabhakaran, S.-K. Mo, Z. X. Shen, Z. Fang, X. Dai, Z. Hussain, and Y. L. Chen, "Discovery of a ThreeDimensional Topological Dirac Semimetal, Na ${ }_{3} \mathrm{Bi}$," Science 343, 864 (2014).

[6] N. P. Armitage, E. J. Mele, and A. Vishwanath, "Weyl and Dirac semimetals in three-dimensional solids," Rev. Mod. Phys. 90, 015001 (2018).

[7] I. Belopolski, S.-Y. Xu, D. S. Sanchez, G. Chang, C. Guo, M. Neupane, H. Zheng, C.-C. Lee, S.-M. Huang, G. Bian, N. Alidoust, T.-R. Chang, B. Wang, X. Zhang, A. Bansil, H.-T. Jeng, H. Lin, S. Jia, and M. Z. Hasan, "Criteria for Directly Detecting Topological Fermi Arcs in Weyl Semimetals," Phys. Rev. Lett. 116, 066802 (2016).

[8] P. J. W. Moll, N. L. Nair, T. Helm, A. C. Potter, I. Kimchi, A. Vishwanath, and J. G. Analytis, "Transport Evidence for Fermi-Arc-Mediated Chirality Transfer in the Dirac Semimetal $\mathrm{Cd}_{3} \mathrm{As}_{2}$," Nature 535, 266 (2016).

[9] D. T. Son and B. Z. Spivak, "Chiral Anomaly and Classical Negative Magnetoresistance of Weyl Metals," Phys. Rev. B 88, 104412 (2013).

[10] Z. Song, J. Zhao, Z. Fang, and X. Dai, "Detecting the Chiral Magnetic Effect by Lattice Dynamics in Weyl
Semimetals," Phys. Rev. B 94, 214306 (2016).

[11] A. Cortijo, D. Kharzeev, K. Landsteiner, and M. A. H. Vozmediano, "Strain-Induced Chiral Magnetic Effect in Weyl Semimetals," Phys. Rev. B 94, 241405 (2016).

[12] A. C. Niemann, J. Gooth, S.-C. Wu, S. Bäßler, P. Sergelius, R. Hühne, B. Rellinghaus, C. Shekhar, V. Süß, M. Schmidt, C. Felser, B. Yan, and K. Nielsch, "Chiral Magnetoresistance in the Weyl Semimetal NbP," Sci. Rep. 7, 43394 (2017).

[13] A. A. Burkov, "Chiral Anomaly and Transport in Weyl Metals," J. Phys. Condens. Matter 27, 113201 (2015).

[14] Y.-Y. Lv, X. Li, B.-B. Zhang, W. Y. Deng, S.-H. Yao, Y. B. Chen, J. Zhou, S.-T. Zhang, M.-H. Lu, L. Zhang, M. Tian, L. Sheng, and Y.-F. Chen, "Experimental Observation of Anisotropic Adler-Bell-Jackiw Anomaly in Type-II Weyl Semimetal WTe ${ }_{1.98}$ Crystals at the Quasiclassical Regime," Phys. Rev. Lett. 118, 096603 (2017).

[15] G. Chang, S.-Y. Xu, H. Zheng, C.-C. Lee, S.-M. Huang, I. Belopolski, D. S. Sanchez, G. Bian, N. Alidoust, T.-R. Chang, C.-H. Hsu, H.-T. Jeng, A. Bansil, H. Lin, and M. Z. Hasan, "Signatures of Fermi Arcs in the Quasiparticle Interferences of the Weyl Semimetals TaAs and NbP," Phys. Rev. Lett. 116, 066601 (2016).

[16] Z. K. Liu, L. X. Yang, Y. Sun, T. Zhang, H. Peng, H. F. Yang, C. Chen, Y. Zhang, Y. F. Guo, D. Prabhakaran, M. Schmidt, Z. Hussain, S. K. Mo, C. Felser, B. Yan, and Y. L. Chen, "Evolution of the Fermi Surface of Weyl Semimetals in the Transition Metal Pnictide Family," Nat. Mater. 15, 27 (2016).

[17] F. Arnold, C. Shekhar, S.-C. Wu, Y. Sun, R. D. dos Reis, N. Kumar, M. Naumann, M. O. Ajeesh, M. Schmidt, A. G. Grushin, J. H. Bardarson, M. Baenitz, D. Sokolov, H. Borrmann, M. Nicklas, C. Felser, E. Hassinger, and B. Yan, "Negative Magnetoresistance without welldefined Chirality in the Weyl Semimetal TaP," Nat. Comm. 7, 11615 (2016).

[18] S.-Y. Xu, I. Belopolski, D. S. Sanchez, C. Zhang, G. Chang, C. Guo, G. Bian, Z. Yuan, H. Lu, Y.-R. Chang, P. P. Shibayev, M. L. Prokopovych, N. Alidoust, H. Zheng, C.-C. Lee, S.-M. Huang, R. Sankar, F. Chou, C.-H. Hsu, H.-T. Jeng, A. Bansil, T. Neupert, V. N. Strocov, H. Lin, S. Jia, and M. Z. Hasan, "Experimental Discovery of a Topological Weyl Semimetal State in TaP," Science Advances 1 (2015).

[19] N. Xu, H. M. Weng, B. Q. Lv, C. E. Matt, J. Park, F. Bisti, V. N. Strocov, D. Gawryluk, E. Pomjakushina, K. Conder, N. C. Plumb, M. Radovic, G. Autès, O. V. Yazyev, Z. Fang, X. Dai, T. Qian, J. Mesot, H. Ding, and M. Shi, "Observation of Weyl Nodes and Fermi Arcs in Tantalum Phosphide," Nat. Comm. 7, 11006 (2016).

[20] S.-M. Huang, S.-Y. Xu, I. Belopolski, C.-C. Lee, G. Chang, B. Wang, N. Alidoust, G. Bian, M. Neupane, C. Zhang, S. Jia, A. Bansil, H. Lin, and M. Z. Hasan, "A Weyl Fermion Semimetal With Surface Fermi Arcs in the Transition Metal Monopnictide TaAs Class," Nat. Comm. 6, 7373 (2015).

[21] H. Weng, C. Fang, Z. Fang, B. A. Bernevig, and X. Dai, "Weyl Semimetal Phase in Noncentrosymmetric Transition-Metal Monophosphides," Phys. Rev. X 5, 011029 (2015).

[22] B. Q. Lv, N. Xu, H. M. Weng, J. Z. Ma, P. Richard, X. C. Huang, L. X. Zhao, G. F. Chen, C. E. Matt, F. Bisti, V. N. Strocov, J. Mesot, Z. Fang, X. Dai, T. Qian, M. Shi, and H. Ding, "Observation of Weyl Nodes in TaAs," Nat. 
Phys. 11, 724 (2015).

[23] B. Q. Lv, H. M. Weng, B. B. Fu, X. P. Wang, H. Miao, J. Ma, P. Richard, X. C. Huang, L. X. Zhao, G. F. Chen, Z. Fang, X. Dai, T. Qian, and H. Ding, "Experimental Discovery of Weyl Semimetal TaAs," Phys. Rev. X 5, 031013 (2015).

[24] L. X. Yang, Z. K. Liu, Y. Sun, H. Peng, H. F. Yang, T. Zhang, B. Zhou, Y. Zhang, Y. F. Guo, M. Rahn, D. Prabhakaran, Z. Hussain, S. K. Mo, C. Felser, B. Yan, and Y. L. Chen, "Weyl Semimetal Phase in the NonCentrosymmetric Compound TaAs," Nat. Phys. 11, 728 (2015).

[25] S.-Y. Xu, I. Belopolski, N. Alidoust, M. Neupane, G. Bian, C. Zhang, R. Sankar, G. Chang, Z. Yuan, C.C. Lee, S.-M. Huang, H. Zheng, J. Ma, D. S. Sanchez, B. Wang, A. Bansil, F. Chou, P. P. Shibayev, H. Lin, S. Jia, and M. Z. Hasan, "Discovery of a Weyl Fermion Semimetal and Topological Fermi Arcs," Science 349, 613 (2015).

[26] S.-Y. Xu, N. Alidoust, I. Belopolski, Z. Yuan, G. Bian, T.-R. Chang, H. Zheng, V. N. Strocov, D. S. Sanchez, G. Chang, C. Zhang, D. Mou, Y. Wu, L. Huang, C.-C. Lee, S.-M. Huang, B. Wang, A. Bansil, H.-T. Jeng, T. Neupert, A. Kaminski, H. Lin, S. Jia, and M. Zahid Hasan, "Discovery of a Weyl Fermion State With Fermi Arcs in Niobium Arsenide," Nat. Phys. 11, 748 (2015).

[27] A. Politano, L. Viti, and M. S. Vitiello, "Optoelectronic Devices, Plasmonics, and Photonics with Topological Insulators," APL Materials 5, 035504 (2017).

[28] J. Hofmann and S. Das Sarma, "Surface Plasmon Polaritons in Topological Weyl Semimetals," Phys. Rev. B 93, 241402 (2016).

[29] H. Yao and T. Shiratsu, "Multipolar Surface Magnetoplasmon Resonances in Triangular Silver Nanoprisms studied by MCD Spectroscopy," J. Phys. Chem. 121, 761 (2017).

[30] Z. Long, Y. Wang, M. Erukhimova, M. Tokman, and A. Belyanin, "Magnetopolaritons in Weyl Semimetals in a Strong Magnetic Field," Phys. Rev. Lett. 120, 037403 (2018).

[31] J. J. Murray, J. B. Taylor, L. D. Calvert, Y. Wang, E. J. Gabe, and J. G. Despault, "Phase Relationships and Thermodynamics of Refractorym Metal Pnictides: The Metal-Rich Tantalum Arsenides," J. Less-Common Met. 46, 311 (1976).

[32] P. Warczok and K. W. Richter, "New Compounds and Phase Equilibria in the System $\mathrm{Hf}-\mathrm{Nb}-\mathrm{As}$," Monatsh. Chem. 143, 1289 (2012).

[33] Z. Li, H. Chen, S. Jin, D. Gan, W. Wang, L. Guo, and X. Chen, "Weyl Semimetal TaAs: Crystal Growth, Morphology, and Thermodynamics," Cryst. Growth Des. 16, 1172 (2016).

[34] B. Da, H. Shinotsuka, H. Yoshikawa, Z. J. Ding, and S. Tanuma, "Extended Mermin Method for Calculating the Electron Inelastic Mean Free Path," Phys. Rev. Lett. 113, 063201 (2014).

[35] D. L. Mills, "The Scattering of Low Energy Electrons by Electric Field Fluctuations Near Crystal Surfaces," Surf. Sci. 48, 59 (1975).

[36] H. Ibach and D. L. Mills, Electron Energy Loss Spectroscopy and Surface Vibrations (Academic Press, San Francisco, 1982).

[37] M. Rocca, "Low-Energy EELS Investigation of Surface Electronic Excitations on Metals," Surf. Sci. Rep. 22, 1
(1995).

[38] C.-C. Lee, S.-Y. Xu, S.-M. Huang, D. S. Sanchez, I. Belopolski, G. Chang, G. Bian, N. Alidoust, H. Zheng, M. Neupane, B. Wang, A. Bansil, M. Z. Hasan, and H. Lin, "Fermi surface interconnectivity and topology in Weyl fermion semimetals TaAs, TaP, NbAs, and NbP," Phys. Rev. B 92, 235104 (2015).

[39] A. B. Sushkov, J. B. Hofmann, G. S. Jenkins, J. Ishikawa, S. Nakatsuji, S. Das Sarma, and H. D. Drew, "Optical Evidence for a Weyl Semimetal State in Pyrochlore $\mathrm{Eu}_{2} \mathrm{Ir}_{2} \mathrm{O}_{7}$," Phys. Rev. B 92, 241108 (2015).

[40] "Phonon and Thermal Expansion Properties in Weyl Semimetals MX $(\mathrm{M}=\mathrm{Nb}, \mathrm{Ta} ; \mathrm{X}=\mathrm{P}, \mathrm{As})$ : ab Initio Studies," Phys. Chem. Chem. Phys. 18, 14503 (2016).

[41] H. W. Liu, P. Richard, Z. D. Song, L. X. Zhao, Z. Fang, G.-F. Chen, and H. Ding, "Raman Study of Lattice Dynamics in the Weyl Semimetal TaAs," Phys. Rev. B 92, 064302 (2015).

[42] F. Arnold, M. Naumann, S.-C. Wu, Y. Sun, M. Schmidt, H. Borrmann, C. Felser, B. Yan, and E. Hassinger, "Chiral Weyl Pockets and Fermi Surface Topology of the Weyl Semimetal TaAs," Phys. Rev. Lett. 117, 146401 (2016).

[43] D. Grassano, F. Bechstedt, and O. Pulci, "Ab initio optical and energy loss spectra of Weyl semimetals TaAs, TaP, NbAs and NbP," arXiv:1804.07505 (2018).

[44] F. de Juan, A. Politano, G. Chiarello, and H. A. Fertig, "Symmetries and Selection Rules in the Measurement of the Phonon Spectrum of Graphene and Related Materials," Carbon 85, 225 (2015).

[45] J. Hofmann and S. Das Sarma, "Plasmon Signature in Dirac-Weyl Liquids," Phys. Rev. B 91, 241108 (2015).

[46] R. E. Throckmorton, J. Hofmann, E. Barnes, and S. Das Sarma, "Many-body effects and ultraviolet renormalization in three-dimensional Dirac materials," Phys. Rev. B 92, 115101 (2015).

[47] R. E. Throckmorton and S. Das Sarma, "Failure of Kohn's theorem and the apparent failure of the $f$-sum rule in intrinsic Dirac-Weyl materials in the presence of a filled Fermi sea," Phys. Rev. B 98, 155112 (2018).

[48] M. Sturaro, E. Della Gaspera, N. Michieli, C. Cantalini, S. M. Emamjomeh, M. Guglielmi, and A. Martucci, "Degenerately Doped Metal Oxide Nanocrystals as Plasmonic and Chemoresistive Gas Sensors," ACS Applied Materials \& Interfaces 8, 30440 (2016).

[49] M. E. Nasir, W. Dickson, G. A. Wurtz, W. P. Wardley, and A. V. Zayats, "Hydrogen Detected by the Naked Eye: Optical Hydrogen Gas Sensors Based on Core/Shell Plasmonic Nanorod Metamaterials," Advanced Materials 26, 3532 (2014).

[50] A. Politano, G. Chiarello, Z. Li, V. Fabio, L. Wang, L. Guo, X. Chen, and D. W. Boukhvalov, "Toward the Effective Exploitation of Topological Phases of Matter in Catalysis: Chemical Reactions at the Surfaces of NbAs and TaAs Weyl Semimetals," Advanced Functional Materials 28, 1800511.

[51] G. C. Silva, F. S. Almeida, M. S. S. Dantas, A. M. Ferreira, and V. S. T. Ciminelli, "Raman and IR spectroscopic investigation of As adsorbed on $\mathrm{Mn}_{3} \mathrm{O}_{4}$ magnetic composites," Spectrochim. Acta, Part A 100, 161 (2013).

[52] S. Sakong and P. Kratzer, "Hydrogen Vibrational Modes on Graphene and Relaxation of the $\mathrm{C}-\mathrm{H}$ Stretch Excitation from First-Principles Calculations," J. Chem. Phys. 133, 054505 (2010).

[53] A. Politano and G. Chiarello, "Periodically Rippled 
Graphene on $\mathrm{Ru}(0001)$ : A Template for Site-Selective Adsorption of Hydrogen Dimers via Water Splitting and Hydrogen-Spillover at Room Temperature," Carbon 61, 412 (2013).

[54] K. Kneipp, Y. Wang, H. Kneipp, L. T. Perelman, I. Itzkan, R. R. Dasari, and M. S. Feld, "Single Molecule Detection Using Surface-Enhanced Raman Scattering (SERS)," Phys. Rev. Lett. 78, 1667 (1997).

[55] J. R. Lakowicz, C. D. Geddes, I. Gryczynski, J. Malicka, Z. Gryczynski, K. Aslan, J. Lukomska, E. Matveeva,
J. Zhang, R. Badugu, and J. Huang, "Advances in Surface-Enhanced Fluorescence," J. Fluorescence 14, 425 (2004).

[56] A. Politano and G. Chiarello, "Quenching of plasmons modes in air-exposed graphene-Ru contacts for plasmonic devices," Applied Physics Letters 102, 201608 (2013).

[57] J. S. Qi, J. Y. Huang, J. Feng, D. N. Shi, and J. Li, "The Possibility of Chemically Inert, Graphene-Based All-Carbon Electronic Devices with 0.8 eV Gap," ACS Nano 5, 3475 (2011). 